\title{
Dynamic Thresholding based Adaptive Canny Edge Detection
}

\author{
Ferdous Hossain \\ Department of Information \& \\ Communication Technology, \\ Mawlana Bhashani Science \& \\ Technology University \\ Tangail, Bangladesh
}

\author{
Mina Asaduzzaman \\ Department of Computer \\ Secience \& Engineering, \\ Dhaka University Of \\ Engineering \& Technology \\ Gazipur, Bangladesh
}

\author{
Mohammad Abu Yousuf \\ Institute of Information \\ Technology(IIT), \\ Jahangirnagar University \\ Dhaka, Bangladesh
}

\author{
Md. Armanur Rahman \\ Department of Computer Secience \& Engineering, \\ Asian University of Bangladesh
}

\begin{abstract}
In this paper, a method for adaptive Canny edge detection algorithm is proposed. Adaptive Canny algorithm is used to increase the accuracy of output objects. In traditional Canny need to set two threshold values manually, so there are some defects to different images but this paper puts faorward an adaptive threshold values based on mean and median values. Our proposed adaptive Canny edge detection method can detect edges successfully which is divided into several steps. First, Gaussian filter is used to smooth and remove noise. Second, gradient magnitude is computed. Third, nonmaximum suppression is applied in which the algorithm removes pixels that are not part of an edge. Finally, hysteresis thresholding is applied which uses two threshold values, upper and lower. A pixel will be marked as an edge if it's gradient lies in between of lower and upper threshold values. A pixel will be discarded if it's gradient below the lower or beyond the upper threshold values. Eventually, the pixels gradient is between the two threshold values will be connected as marked edge. The experimental results show the efficacy of the proposed method.
\end{abstract}

\section{General Terms}

Noise Reduction of Images, Compute Gradient Magnitude and Angle, Non-Maximum Suppression, Hysteresis Thresholding, Adaptive Canny Edge Detection Algorithms

\section{Keywords}

Adaptive canny; sobel; dynamic threshold; edge detection

\section{INTRODUCTION}

Edge detection is a crucial part in object recognition. It is a process of finding sharp discontinuities in an image. The discontinuities are abrupt changes in pixel intensity which characterize boundaries of objects in a scene. In short, the goal of edge detection is to produce a line drawing of the input image. The extracted features are then used by computer vision algorithms, e.g. recognition and tracking [1]. A classical method of edge detection involves the use of operators, a two dimensional filter. An edge in an image occurs when the gradient is greatest. The operator works by identifying these large gradients to find the edges. There are different types of operators designed to detect certain types of edges. The operators can be configured to search for vertical, horizontal, or diagonal edges. Major problem to detect edge is noise. It is not enough to simply reduce the noise, because the image will be either distorted or blurred. Adaptive Canny operator has enough data to discount localized noisy pixels. However, the change in intensity is not always a step change. The intensity change can also be continuous where the operator then has to be modified for proper edge detection. Consequently, there are problems of missing true edges, false edge detection, and high computational time. In this paper, adaptive Canny edge detection algorithm is developed where threshold values are dynamically defined based on mean and median values. Adaptive Canny edge detector performs necessary operation to evaluate adaptive Canny edge detection algorithm to detect edges in $\mathrm{X}$ and $\mathrm{Y}$ direction. The remainder of the paper is organized as follows. The proposed adaptive Canny edge detection method is described in section II. Evaluation criteria are explained in section III and experimental results are placed in section IV. Finally, mention conclusion in the last section.

\section{PROPOSED ADAPTIVE CANNY EDGE DETECTION METHOD}

In this paper, a new edge detection method known as adaptive Canny edge detection method is proposed. It is a multi-step process, which can be implemented on the central processing unit (CPU) as a sequence of filters [2]. The proposed edge detection method consists of the following steps. To describe all the steps required to detect edges, the images shown in Fig. 1 are considered.

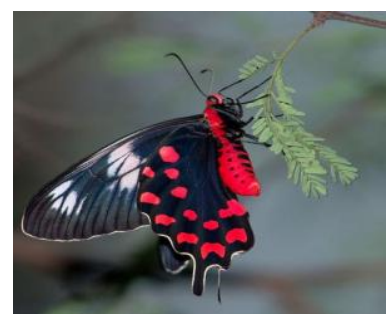

a) Butterfly

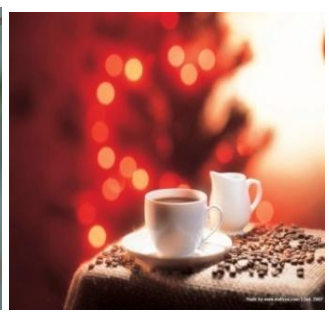

b) Coffee Cup 


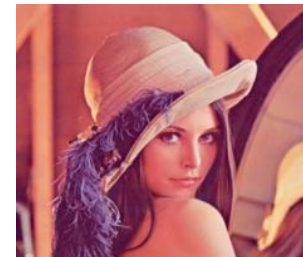

c) Lena

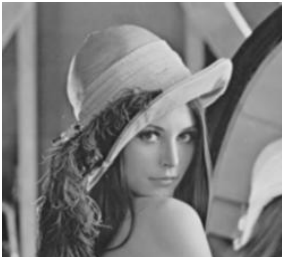

d) Lena-1
Fig. 1. Original images

\subsection{Convert to Gray Scale Image and Noise \\ Reduction}

We first convert the color images into gray scale images [3]. Noise is removed from the gray scale images after the conversion. Usually noise reduction implies some sort of blurring operation. Here, Gaussian filter is used for noise reduction. $5 \times 5$ filter as shown in Fig. 2 is specially used for more accuracy. Note that this $5 \times 5$ filter is equivalent to the Gaussian filter. Images after noise reduction are shown in Fig.

\begin{tabular}{|c|c|c|c|c|c|}
\hline \multirow{2}{*}{1} & 2 & 4 & 5 & 4 & 2 \\
\hline & 4 & 9 & 12 & 9 & 4 \\
\hline \multirow{3}{*}{15} & 5 & 12 & 15 & 12 & 5 \\
\hline & 4 & 9 & 12 & 9 & 4 \\
\hline & 2 & 4 & 5 & 4 & 2 \\
\hline
\end{tabular}

Fig. 2. Gaussian filter

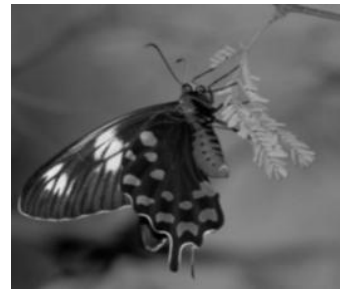

a) Butterfly

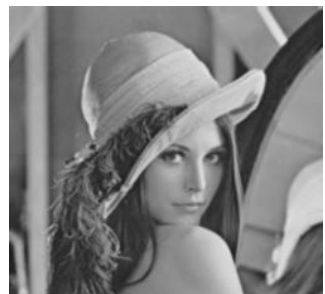

c) Lena

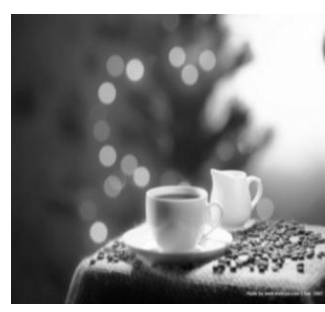

b) Coffee Cup

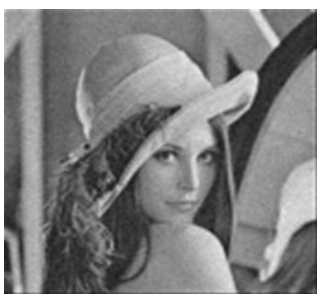

d) Lena-1
Fig. 3. Gray scale images after noise reduction

\subsection{Compute Gradient Magnitude and Angle}

Here, we calculate the vertical and horizontal gradients as shown in Fig. 4. used convolution kernels [4]. This is input to the magnitude calculation unit which computes, at each pixel location, the gradient magnitude (D) from the pixel's horizontal and vertical gradients by using equation (1).

$D=\sqrt{G_{x}^{2}+G_{y}^{2}}$

The angle of the gradient is calculated by using equation (2).

$\theta=\arctan \left(\frac{G_{x}}{G_{y}}\right)$
The gradient images are shown in Fig. 5.

\begin{tabular}{|l|l|l|}
\hline-1 & 0 & +1 \\
\hline-2 & 0 & +2 \\
\hline-1 & 0 & +1 \\
\hline
\end{tabular}

\begin{tabular}{|c|c|c|}
\hline+1 & +2 & +1 \\
\hline 0 & 0 & 0 \\
\hline-1 & -2 & -2 \\
\hline
\end{tabular}

Fig. 4. Horizontal and vertical gradient

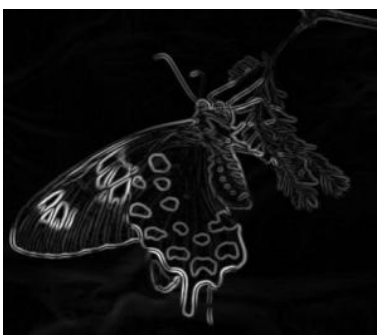

a) Butterfly

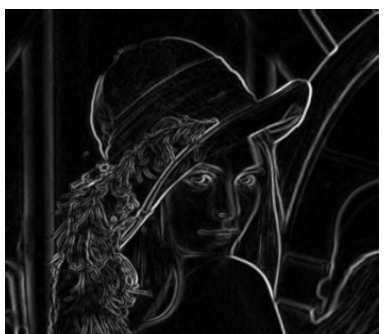

c) Lena

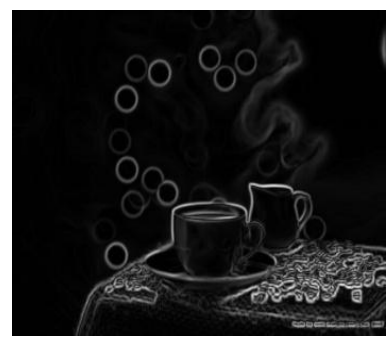

b) Cofee Cup

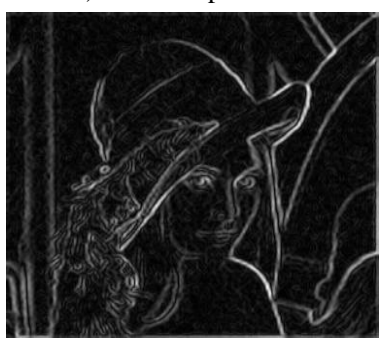

d) Lena-1
Fig. 5. Gradient images

\subsection{Non-Maximum Suppression}

Actually when we used the Sober filter, the edges it finds can be either very thick or very narrow depending on the intensity across the edge and how much the image was blurred first. One would like to have edges that are only one pixel wide. The non-maximal suppression step keeps only those pixels on an edge with the highest gradient magnitude. These maximal magnitudes should occur right at the edge boundary, and the gradient magnitude should fall off with distance from the edge. So, widely used three pixels in a $3 \times 3$ around pixel (x, $\mathrm{y})$ are examined. If pixel $(\mathrm{x}, \mathrm{y})$ has the highest gradient magnitude of the three pixels examined, it is kept as an edge. If one of the other two pixels has a higher gradient magnitude then pixel $(\mathrm{x}, \mathrm{y})$ is not on the center of the edge and should not be classified as an edge pixel. The images after nonmaximum suppression are shown in Fig. 6.

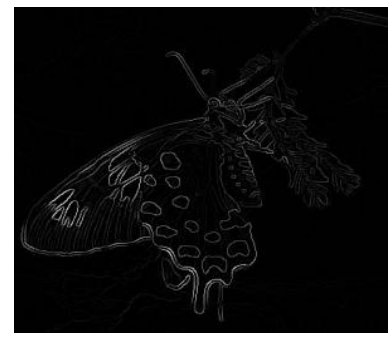

a) Butterfly

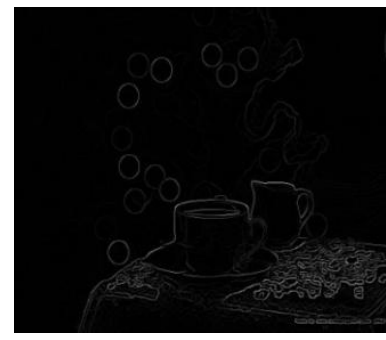

b) Coffee Cup 


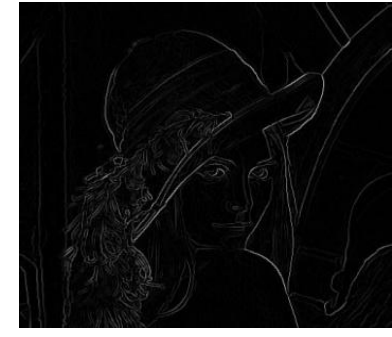

c) Lena

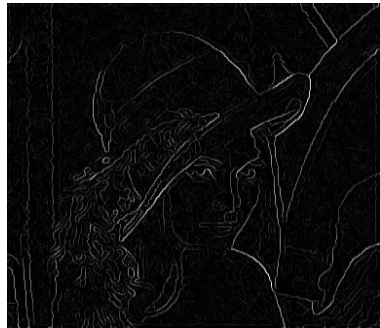

d) Lena-1
Fig. 6. Non-maximum suppressed images

\subsection{Hysteresis Thresholding of Adaptive Canny}

Some of the edges detected by steps A-C will not actually be valid, but just be noise. We would like to filter this noise out. Eliminating pixels whose gradient magnitude D falls below some threshold removes the worst of this problem, but it introduces a new problem. A simple threshold may actually remove valid parts of a connected edge, leaving a disconnected final edge image. This happens in regions where the edge's gradient magnitude fluctuates between just above and just below the threshold. Hysteresis is one way of solving this problem. Instead of choosing a single threshold, two thresholds $t_{\text {high }}$ and $t_{\text {low }}$ are used. The adaptive upper and the lower threshold are determined [5, 6, 7, 8,9,10] by using equation (3) and (4):

$$
\begin{gathered}
t_{\text {low }}=\max (0,(\mu-\sigma) / a) \\
t_{\text {high }}=\min (A,(\mu+\sigma) / b)
\end{gathered}
$$

Where, $\mu$ is the mean value of the normalized probability density function,

$\sigma$ is the variance of the probability density function,

$a$ is a parameter between 5 and 7 , preferably 6 ,

$b$ is a parameter between 2 and 4 , preferably 3 , and

$A$ is the maximum intensity value.

Pixels with a gradient magnitude $D<t_{\text {low }}$ or $D>t_{\text {high }}$ are discarded immediately. However, pixels with gradient magnitude $D \geq t_{\text {low }}$ and $D \leq t_{\text {high }}$ are only kept if they form a continuous edge line with pixels with high gradient magnitude (i.e., above $t_{\text {high }}$ ).

Final edge images after step D is demonstrated in Fig. 7.

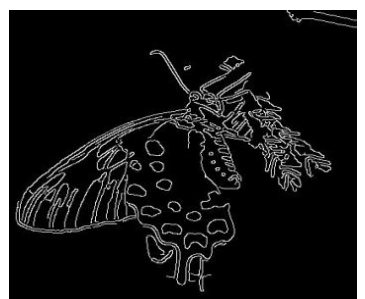

a) Butterfly

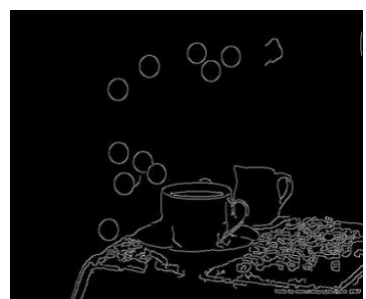

b) Coffee Cup

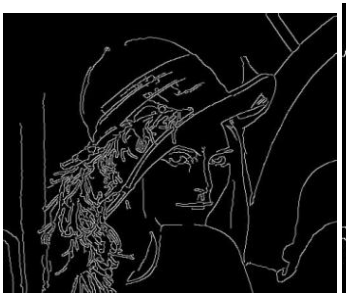

c) Lena

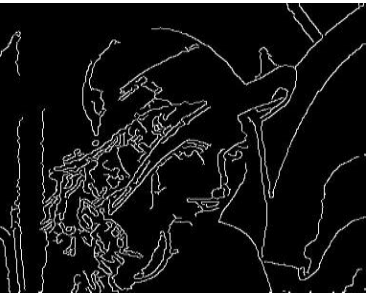

d) Lena-1
Fig. 7. Final result images

Work flow of the proposed adaptive Canny Edge detection method is shown in Fig. 8.

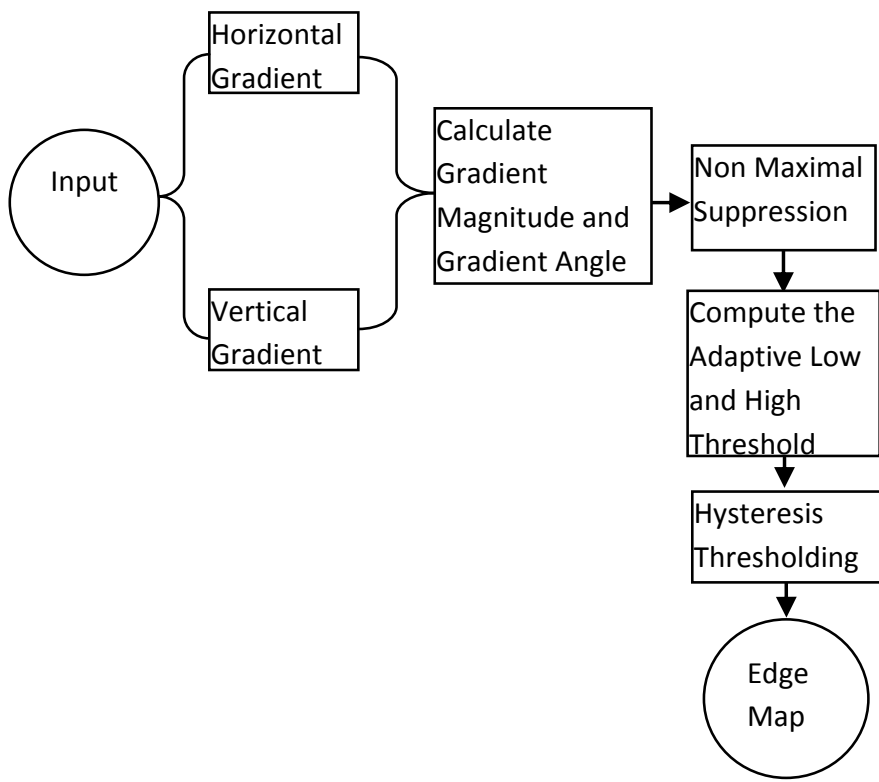

Fig. 8. Basic steps of adaptive Canny algorithm

\section{EVALUATION CRITERIA}

To evaluate the performance of the proposed adaptive Canny Edge detection method Signal to Noise Ratio (SNR), Mean Square Error (MSE) and Edge Preservation Factor (EPF) parameter are used [11].

Signal to Noise Ratio (SNR):

$S N R=-10 \log 10\left[\frac{\sum_{x=1}^{M} \sum_{y=1}^{N}\left(I_{d}(x, y)-I(x, y)\right)^{2}}{\sum_{x=1}^{M} \sum_{y=1}^{N}\left(I_{d}(x, y)\right)^{2}}\right]$

Mean Square Error (MSE):

$$
M S E=\left[\frac{1}{M \cdot N} \sum_{x=0}^{M-1} \sum_{y=0}^{N-1}\left(I(x, y)-I_{d}(x, y)\right)^{2}\right]
$$

Where, the image size is $M \times N$. $x$ means row, y means column, $I$ means original image and $I_{d}$ means filtered Image.

Edge Preservation Factor (EPF):

$$
E P F=\frac{\sum(\Delta I-\overline{\Delta I})\left(\Delta I_{d}-\overline{\Delta I_{d}}\right)}{\sqrt{\sum(\Delta I-\overline{\Delta I})^{2}\left(\Delta I_{d}-\overline{\Delta I_{d}}\right)^{2}}}
$$

Where $\Delta I$ and $\Delta I_{d}$ are the high pass filtered versions of Images $I$ and $I_{d}$ obtained with a $3 \times 3$ pixel standard approximation of the Laplacian operator. The larger value of EPF means more ability to preserve edges 


\section{EXPERIMENTAL RESULTS}

The proposed algorithm has been applied to $2 \mathrm{D}$ image with have been corrupted by noise. The computation is carried out on C\#.Net in visual studio 2008 in a Core i5 $1.8 \mathrm{GHz}$ and 4GB RAM laptop having a Windows 7 Home Premium operating system. Experiment was done on hundred of images but here four images in 512*512 sizes (e.g. Butterfly, Coffee Cup, Lena, and Lena-1) are mentioned for testing the performance of the proposed algorithm. Moreover, the performance of our proposed method is compared with the Sobel and traditional Canny edge detection method. Table 1 shows SNR, EPF and MSE values of four images (Butterfly, Coffee Cup, Lena, and Lena-1 images) with respect Sobel, traditional Canny and proposed (Adaptive Canny) Methods. Fig. 9 shows comparison graph of SNR with respect of applied methods where it shows that SNR are highest for all four images for the case of proposed method among the all methods.

Table 1. SNR, EPF AND MSE values of experimental images

\begin{tabular}{|c|c|c|c|c|c|c|c|c|c|}
\hline $\begin{array}{c}\text { Operato } \\
\mathrm{r}\end{array}$ & \multicolumn{3}{|c|}{ Sobel } & \multicolumn{3}{c|}{ Canny } & \multicolumn{2}{|c|}{ Proposed (Adaptive Canny) } \\
\cline { 2 - 10 } & SNR & EPF & MSE & SNR & EPF & MSE & SNR & EPF & MSE \\
Image & & & & & & & & & \\
\hline Butterfly & $\begin{array}{c}17.45 \\
6\end{array}$ & $\begin{array}{c}0.41 \\
5\end{array}$ & $\begin{array}{c}6.43 \\
5\end{array}$ & $\begin{array}{c}18.17 \\
5\end{array}$ & $\begin{array}{c}0.52 \\
7\end{array}$ & $\begin{array}{c}6.63 \\
2\end{array}$ & $\begin{array}{c}19.91 \\
6\end{array}$ & $\begin{array}{c}0.65 \\
5\end{array}$ & $\begin{array}{c}5.12 \\
3\end{array}$ \\
\hline $\begin{array}{c}\text { Coffee } \\
\text { Cup }\end{array}$ & $\begin{array}{c}17.87 \\
3\end{array}$ & $\begin{array}{c}0.39 \\
1\end{array}$ & $\begin{array}{c}6.33 \\
2\end{array}$ & $\begin{array}{c}18.19 \\
2\end{array}$ & $\begin{array}{c}0.43 \\
9\end{array}$ & $\begin{array}{c}6.10 \\
8\end{array}$ & $\begin{array}{c}19.99 \\
8\end{array}$ & $\begin{array}{c}0.53 \\
2\end{array}$ & $\begin{array}{c}5.00 \\
8\end{array}$ \\
\hline Lena & $\begin{array}{c}22.40 \\
4\end{array}$ & $\begin{array}{c}0.45 \\
3\end{array}$ & $\begin{array}{c}5.30 \\
4\end{array}$ & $\begin{array}{c}22.52 \\
3\end{array}$ & $\begin{array}{c}0.53 \\
2\end{array}$ & $\begin{array}{c}4.80 \\
8\end{array}$ & $\begin{array}{c}23.87 \\
2\end{array}$ & $\begin{array}{c}0.68 \\
1\end{array}$ & $\begin{array}{c}4.13 \\
6\end{array}$ \\
\hline Lena-1 & $\begin{array}{c}22.56 \\
7\end{array}$ & $\begin{array}{c}0.48 \\
2\end{array}$ & $\begin{array}{c}5.31 \\
2\end{array}$ & $\begin{array}{c}22.63 \\
2\end{array}$ & $\begin{array}{c}0.54 \\
8\end{array}$ & $\begin{array}{c}4.85 \\
8\end{array}$ & $\begin{array}{c}23.91 \\
4\end{array}$ & $\begin{array}{c}0.68 \\
7\end{array}$ & $\begin{array}{c}4.13 \\
5\end{array}$ \\
\hline
\end{tabular}

Fig. 10 show the larger value of EPF means more ability to preserve edges. Finally, Fig. 11 shows the result of MSE, where MSE are lowest for the case of proposed method.

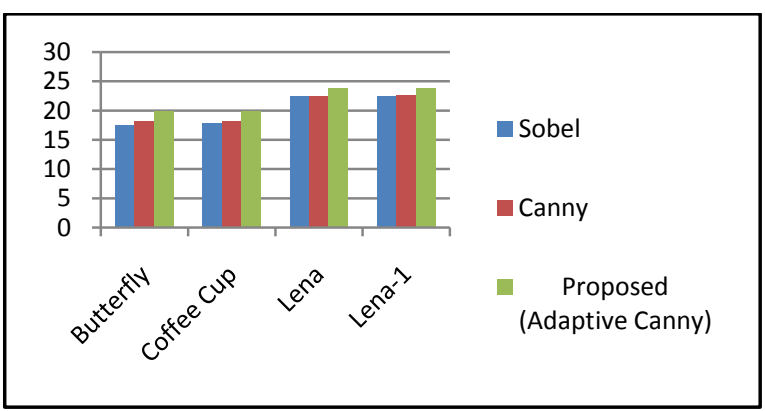

Fig. 9. Comparison of SNR of experimental images.

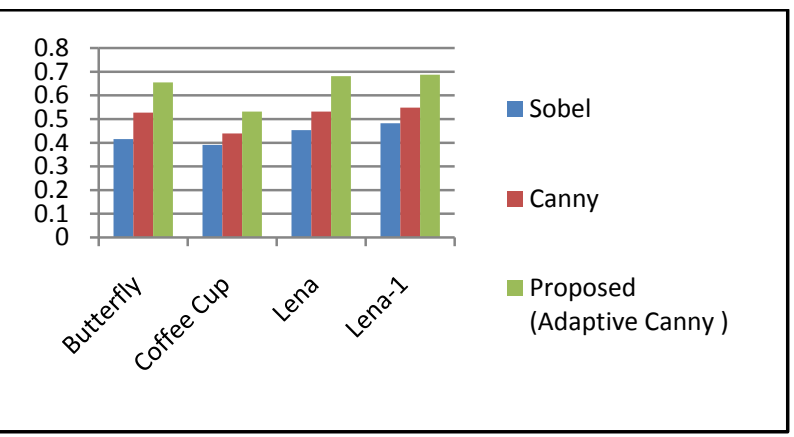

Fig. 10. Comparison of EPF of experimental images.

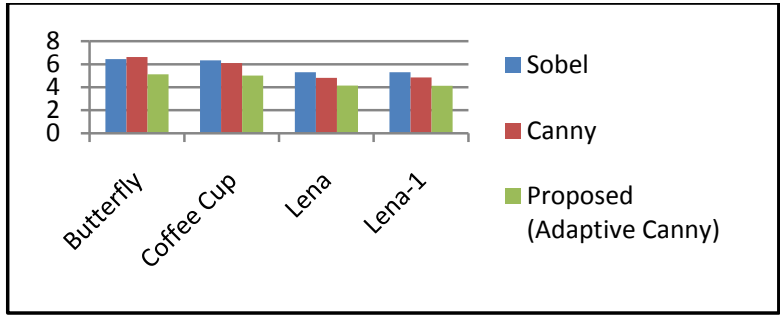

Fig. 11. Comparison of MSE of experimental images.

\section{CONCLUSION}

In this paper an effective method for edge detection via dynamic thresholds of adaptive Canny algorithms. The edge detection is the primary task in identifying an object from an image, it is vitally important to know the advantages and disadvantages of each edge detection filters. This is a novel edge-detection algorithm is to provide an errorless solution that is adaptable to the different noise levels of these images to help in identifying the valid image contents. This work has been done for the edge detection techniques of the Gradientbased. The edge detection techniques are compared with case study of identifying a different type of image. The software was implemented using C\#. Gradient-based algorithms have major drawbacks in sensitive to noise. Adaptive Canny operator can be applied to different situations for detect the edge clearly. In order to improve its performance, we propose the calculation of gradient magnitude and gradient direction based on 8 neighborhoods, and makes the adaptive calculation of the threshold of adaptive Canny operator. The experimental results show that the edge detected by the improved Canny operator has more continuity, and greater signal to noise ratio.

\section{REFERENCES}

[1] Sohag Kabir and A S M Ashraful Alam.2014."Hardware Design and Simulation of Sobel Edge Detection Algorithm”. Image, Graphics and Signal Processing, 5, 10-18.

[2] Mr. Manoj K.Vairalkar and Prof. S.U.Nimbhorkar.2012 "Edge Detection of Images Using Sobel Operator". International Journal of Emerging Technology and Advanced Engineering ,ISSN 2250-2459, Volume 2, Issue 1

[3] O.R. Vincent and O. Folorunso. 2009. A Descriptive Algorithm for Sobel Image Edge Detection.Proceedings of Informing Science \& IT Education Conference (InSITE)

[4] T.Rupalatha1,Mr.C.Leelamohan2,Mrs.M.Sreelakshmi3.2 013."IMPLEMENTATION OF DISTRIBUTED CANNY EDGE DETECTOR ON FPGA" International Journal of Innovative Research in Science, Engineering and Technology Vol. 3, Issue5,

[5] T.Rupalatha1,G.Rajesh2,K.Nandakumar3.2013.“Implem entation of Distributed Canny Edge Detector on FPGA “ International Journal of Computer Engineering Science (IJCES) Volume 3 Issue 5 (May 2013 Vol. 2, Issue7,

[6] Yuan-Kai Huo, Gen Wei, Yu-Dong Zhang and Le-Nan Wu. 2010. "An Adaptive Threshold for the Canny Operatorof Edge Detection". IEEE 978-1-4244-5555$3 / 10 / \$ 26.00$

[7] Ping ZHOU1, Wenjun YE1, Yaojie XIA1, Qi WANG2. 2011. "An Improved Canny Algorithm for Edge 
Detection". Journal of Computational Information Systems

[8] Y.L.Zhang and L.Yan.2009. "Edge Detection Base on Adaptive Canny Method ". M.Sc. thesis, Norstwest University, Xi'an, China, Jun

[9] Koba Natroshvili, Ayyappan Mani .2013."EDGE DETECTION WITH ADAPTIVE THRESHOLD ," US $8,391,612$ B2.
[10] Ferdous Hossain, Mithun Kumar P.K., Mohammad Abu Yousuf. 2015. "Hardware Design and Implementation of Adaptive Canny Edge Detection Algorithm International Journal of Computer Applications, Vol. 124, No. 9

[11] Mohammad Motiur Rahman. Mithun Kumar PK and Mohammad Shorif Uddin.2014. "Optimum Threshold Parameter Estimation of Wavelet Coefficients Using Fisher Discriminant Analysis for Speckle Noise Reduction," The International Arab Journal of Information Technology, Vol. 11, No. 6 